\title{
Beiträge zur Histologie der weichen Naevi.")
}

(Ein Fall von spitzem Condylom am kleinen Finger der rechten Hand, xanthomartigen Naevus verrucosus der rechten Achselhöhle und mehreren über den Körper zerstreuten Naevi lineares. ${ }^{* *}$ )

Von

Dr. Otto Sachs,

Sekundararzt des k. k. allgem. Krankenhauses in Wien, friherem Assistenten der Klinik.

(Hiezu Taf. VII u. VIII.)

Der vorliegende Fall bietet nicht nur in klinischer, sondern auch histologischer liichtung eine Reihe bemerkenswerter Tatsachen, deren Veröffentlichung geeignet erscheint, besonders die II istologie der weichen Naevi in mancher Beziehung zu vervollständigen.

Aus der Krankengeschichte des 8jährigen Mädchens, das ich in der Breslauer dermatol. Klinik zu beobachten Gelegenheit hatte, sei folgendes angeführt.

Bei dem aus gesunder Familie stammenden Kinde handelt es sich um eine kongenitale Affektion.

Eltern, sowie fünf Geschwister der Patientin sind gesund, eine hereditäre Belastung ist nicht nachweisbar. In der Familie bestehen keine Erkrankungen innerer Organe, speziell von seiten der Leber oder solche nervöser Natur.

*) Die klinische wie histologische Bearbeitung des Falles wurde in der Breslauer dermat. Univ.-Klinik des Herrn Prof. A. N eisser begonnen and im patholog.-bistolog. Institute des Herrn Prof. R. Paltauf in Wien beendigt.

**) Demoxstration dieses Falles in der Breslauer dermatolog. Vereinjgung am 26. Oktober 1901. Dieses Archiv Bd. LX. 1902.

Demonstration mikroskopiseher Präparate dieses Falles in der Wiener dermatolog. Gesellschaft am 11. Februar 1903. 
Nach den Angaben der Mutter dieses Mädchens wurde die Erkrankung bald nach der Geburt bemerkt und begann am rechten Handgelenk mit einer "kleinen Blatter", von da zog sich dann "ein Streifen ${ }^{\mu}$ bis an die Endphalanx des kleinen Fingers und nach und nach auch an die anderen Finger derselben Hand. Zuerst waren ndie Grinde trocken, später eiterten dieselben.

Gleichzeitig wurde "die rechte Achselhöble wund", doch breitete sich trotz Anwendung von Streupulvern diese "wunde Fläche" über den halben Bauch und Hals aus, sowie den rechten Arm in Form von Streifen.

Diese Stellen heilten bis auf die Affektion in der rechten Achselböhle und an den Fingern aus. An den Fingern der linken Hand, ebenso an den Zehen, sowie am Gesäße traten mit "trockenen Grinden" versehene Streifen auf.

Das Mädchen ist entsprechend seinem Alter gut entwickelt, nur erscheint die Muskulatur der rechten oberen Extremität im Vergleich mit der linken oberen etwas atrophisch, sie wird größtenteils mit der linken Hand gestätzt, ihre motorische Kraft ist etwas geringer als die der linken. Andere nervöse Störungen wurden nicht konstatiert.

Die Untersuchung der inneren Organe, der Schleimhaut des Mundes und Rachens ergab nichts pathologisches, ebensowenig die des Blutes und Urins.

Auf der Dorsalseite der Endplalange des kleinen Fingers der rechten Hand findet sich eine Geschwulst von der Größe, Aussehen und Farbe einer Himbeere, welche der Unterlage fest aufsitzt. Ähnliche Exkreszenzen, etwa hanfkorngroß, finden sich auch an den Endphalangen derselben Hand.

Die Durchleuchtung dieser mittelst Röntgenstrablen ergab ein völliges Intaktsein sämtlicher Phalangealknochen.

Die erkrankte Fläche der rechten Achselhöhle hat eine rosarote Farbe, ist gewulstet und ödematös, so da 1 die normalen Hautfalten dieser Gegend sich als tiefe Furchen markieren.

Die infiltrierte, ödematöse Haut fühlt sich bei Palpation ziemlich weich an und läßt sich leicht in Falten abheben, in diesen und auf der Fläche selbst ist stets reichliches Sekret vorhanden.

Schon makroskopisch kann man einen verrucösen Bau deutlich erkennen, so daß die ganze Fläche ein sammetartiges Aussehen erhält, dabe nur wenig das Niveau der umgebenden gesunden Hautpartien überragt.

Die Umgebung dieser infiltrierten und ödematösen Haut ist etwa $2 \mathrm{~cm}$ ringsherum gerötet, teils nässend, teils schuppend.

Mit verhornten Massen bedeckte, strichförmig verlaufende, 4-5 cm lange $\mathrm{Naevi}$ lineares befinden sich an der Ulnarseite der I. Phalangedes Mittelfingers der rechten Hand, der Radialseiteder II. Phalange des Zeigefingers und der Ulnarseite der I. Phalange des kleinen Fingers der linken Hand, der rechten Glutealgegend und einzelnen Zehen des rechten $\mathrm{Fu}$ us. 
Die Therapie des himbeerförmigen Tumors am kleinen Finger der rechten Hand bestand darin, daß derselbe mit dem Skalpell entfernt, die Wundfläche mit dem Paquelin verschorft wurde. Die hanfkorngroßen Exkreszenzen an den übrigen Fingern wurden mit dem scharfen Löffel ausgekratzt. An den Fingern blieb noch lange Zeit ein geringer Grad von Entzündungserscheinungen bestehen.

Beim Naevus der Achselhöhle wurden anfangs zur Beseitigung der starken Sekretion extern Pasten und austrocknende Streupulver, zur Zerstörung der Papillen der Paquelin mit geringem Erfolge angewendet.

Die interne Darreichung von Thyreoidtabletten nach dem Vorschlage von C. Beck hatte ebenfalls keine Besserung erzielt.

Diese geringe Aussicht auf Besserung des Leidens gab die Veranlassung zur totalen Excision des Naevus, die in Chloroformnarkose in der Breslauer chirurgischen Klinik von Herrn Prof. Henle mit gleichzeitiger Exstirpation einiger vergrößerter Lymphdrüsen der betreffenden Achselhöhle ausgeführt wurde.

Die Wunde heilte reaktionslos per primam.

Die an einzelnen Fingern beider Hände, der rechten Glutaealgegend und an einzelnen Zehen des rechten Fußes befindlichen $\mathrm{Naevi}$ lineares wurden anfangs durch Applikation eines 5\% Salizyl-Seifenpflasters zum Erweichen versucht, nachher ebenfalls mit dem scharfen Löffel ausgekratzt.

Die rom kleinen Finger der rechten Hand, den übrigen Fingern, sowie der rechten Achselhöhle excidierten Stücke wurden teils in Alkohol und reiner Osmiumsäure gehärtet, teils in Formol, Müller, Zenker, Sublimat und Flemming fixiert und in steigendem Alkohol nachgehärtet.

Die Einbettung geschah sowohl in Paraffin, wie Celloidin.

Als Kern- und Protoplasmafärbungen kamen Hämatoxylin, Hämalaun, Eosin, polychromes Methylenblau (Entfärbung mit Glyzerin-Äther-Mischung), Indulin, Vesuvin, Boraxkarmin, Pappenheims Methode mit Methylgrün-Pyronin in Verwendung.

Das Bindegewebe wurde nach van Gieson tingiert; auf elastische Fasern wurde nach Weigert, TaenzerUnna und Pranter, Protoplasmafasern nach den Angaben von Kromayer und Unna gefärbt.

Zum Nachweis des Fettes diente die Osmiumsäure; außerdem wurden an Gefrierschnitten, als auch gehärteten 
Schnitten das von Da d d i zur Fettfärbung empfohlene S ud a n III. angewendet.

Die histologische Untersuchung eines Längsschnittes durch den himbeerförmigen Tumor am kleinen Finger der rechten Hand bietet folgendes Bild.

In den handschuhfingerförmig verzweigten Papillen sind die Gefäße mäßig erweitert. Im Anschlusse an diese, sowie auch im papillären und subpapillären Bindegewebe liegen vereinzelte oder in kleinen Gruppen angeordnete Infiltrationszellen, welche sich aus poly- oder mononucleären Leucozyten zusammensetzen.

In den Papillen sieht man sowobl Mastzellen (Färbung mit polychromem Methylenblau oder Pyronin-Methylgrün nach P a p p e nhei m), als auch Plasmazellen, in manchen Papillen mehr, in manchen wieder weniger.

Die Mastzellen sind meistens an der Peripherie der Papillen gelagert, einzelne von ihnen dringen bis an die Basalzellenschichte vor. In der Stachel- und Basalzellenschichte selbst waren weder Mast- noch Plasmazellen nachweisbar.

Die Plasmazellen finden sich wiederum mehr in der Nähe der Gefäße zu kleinen Gruppen angeordnet.

Die Gefä be selbst sind beträchtlich erweitert, die Wandungen ein wenig verdickt, an einzelnen Stellen derselben sieht man polynucleäre Lencozyten, einige in Auswanderung begriffen.

Das Lumen dieser erweiterten Gefäße ist mit noch gut erhaltenen roten Blutkörperchen und vereinzelten polynucleären Leucozyten ausgefüllt.

Das Bindegewebe der Papillen sowie der subpapillären Schichte zeigt ein lockeres Gefüge. Die einzelnen Bündel sind durch seröse Durchtränkung weit auseinander gedrängt.

Die elastischen Fasern sind gut erhalten und verlaufen nur in der subpapillären Schichte und ziehen nicht in die einzelnen Papillen des Tumors hinauf.

Die Zellen der Epidermis sind nur herdweise auseinander gedrängt. Schon bei schwacher Vergrößerung (Übersichtsfärbung mit Hämalaun-Eosin, Hämatoxylin Eosin, van Gieson) bemerkt man, daß an diesen Stellen weite Spalträume die einzelnen Zellen von einander getrennt balten.

In diesen Zwischenräumen lagern sich sowohl mono- als auch polynucleäre Leucozyten ein. Letztere finden sich aber auch an anderen Stellen der Epidermis vereinzelt und gruppiert, teils auf, teils zwischen den Zellen oder die Basalzellenschichte durchdringend.

Dieser Befund deutet auf einen bestehenden Entzündungsprozeß hin, der seine Frklärung in dem anhaltenden Reiz durch Sekrete findet. 
Ein schmaler Saum des Stratum granulosum, dessen Zellen vereinzelt einen gut färbbaren Kern aufweisen, bedeckt die Stachelzellenschicht, welche ihre großen, verdickten und verzweigten Zapfen in die Coriumschichte sendet.

Die Stachelzellen und Interspinalspalten zeichnen sich durch ihre beträchtliche Größe, sowie die zahlreichen und andauernd vorhandenen Mitosen auch in den höheren Lagen der Stachelschicht aus.

Bei Anwendung von starken Vergrößerungslinsen (Zeiß: ÖlImmersion, Okul. 2) werden bei dünnen Schnitten (Paraffin $3-5 \mu$ ) und Färbung mit Hämalaun-Eosin, noch deutlicher nach der Kromayerschen Färbung die Epithelfasern zur Anschaung gebracht.

Die Epithelfasern sind in allen Schichten der Epidermis deutlich erhalten, erleiden nur an einzelnen Stellen eine Unterbrechung.

An Querschnitten läßt sich genau die Zusammensetzung des Tumors erkennen.

Die dem himbeerförmigen Aussehen des Tumors entsprechenden Konturen verlaufen in konvexen Linien. Eine solche entspricht der Oberfläche eines einzelnen Zapfens, welcher seitlich geradlinig begrenzt, gegen die Basis des Tumors konvergierend verläuft. Zwischen diesen einzelnen Zapfen befindet sich eine Bindegewebsschichte, welche als Bindeglied die Zapfen fächerförmig zu dem himbeerförmigen Tumor vereinigt.

Der histologische Bau dieser Affektion gleicht sowohl seiner Konfiguration als auch seiner Struktur nach, vollkommen dem eines spitzen Kondyloms.

Die Affektion in der rechten Achselhöhle ist durch seine kleinen, makroskopisch schon sichtbaren, sammetartig sich antühlenden, rosarot gefärbten Papillen ausgezeichnet, welche mikroskopisch einen deutlich verrucösen Bau zeigen.

Auf den Schnitten sind die Papillen in verschiedener Richtung getroffen, man sieht neben längs und schräg, eine ganze Reihe von quer getroffenen. Diese verschiedeuartig verzweigten, mälig verdickten, dem verrucösen Bau entsprechend verschieden langen interpapillären Retezapfen senken sich in die Tiefe der Coriumschichte ein. Gegen die umgebende gesunde Haut nehmen diese interpapillären Retezapfen an Länge und Breite immer mehr ab.

Diejenigen Schnitte, die in reiner $1 \%$ Osmiumsäure fixiert und gehärtet warden, lassen schon bei mittelstarker Vergrößerung an ungefärbten Präparaten in den Papillen sowie im subpapillären Anteil der Cutis Zellen erkennen, die jedenfalls nicht zum gewöhnlichen Bilde eines weichen und nicht pigmentierten Naevus gehören. 
Die teils elliptisch, teils rhombisch geformten Zellen sind ausgefüllt von dichtgedrängten, fast gleichgroßen, durch Osmiumsäure schwarz gefärbten Tröpfchen, ebenso schwarz gefärbt wie das subkutane Fettgewebe.

Die Zellen enthalten einen, oder auch zwei Kerne, welche bei manchen zentral, bei anderen wiederum exzentrisch gelagert sind.

Der Kontur des Kernes ist gegen das umgebende Protoplasma bei Alkoholhärtung und Färbung mit polychromem Methylenblau durch einen blauen Rand deutlich abgegrenzt. Das Kerngerüst tritt deutlich zu Tage und in demselben sieht man 1-2, öfters auch mehrere dunkelblau gefärbte Kernkörperchen. Manche dieser Zellen zeigen einen geschrumpften Kern.

Das Bild zahlreicher Kerne entsteht dadurch, daß diese durch das zarte Protoplasma darüber gelagerter Zellen durchschimmern, so daß man in dem Rahmen einer Zelle oft die Kerne einer ganzen Anzahl übereinander gelagerter Zellen erblickt. Ein genaues Einstellen läßt jedoch die verschiedenen dazu gehörenden Zellkonturen leicht erkennen.

Riesenzellen waren in den Präparaten nicht zu finden.

Die Zellen erinnern in ibrer Form, Größe und Bau an Xanthom. zellen, mit denen sie eine gewisse Ähnlichkeit aufweisen.

D i ese „xanthomähnlichen Zellen"füllen fast die ganze Papille, ebenso einen Teil des subpapillären Bindegewebes aus. Von der Basalzellenschichte sind sie größtenteils durch einen schmalen Saum lockeren Bindegewebes geschieden, an anderen Stellen reichen dieselben bis zu den Basalzellen dicht heran.

In einzelnen Papillen liegen sie ganz enge aneinander, ohne daß man auch nur den kleinsten Raum zwischen ihnen sehen kann. In anderen wiederum sind dieselben nur in einem losen Zusammenhange und lassen weite Lücken untereinander frei.

Da aber auch gerbstoff haltige Elemente von Osmiumsäure geschwärzt werden, mußten jene Methoden in Anwendung gebracht werden, welche in überzeugender Weise die Fettreaktion zeigen.

$\mathrm{Zu}$ diesem Zwecke wurden zunächst Gefrierschnitte, nachher auch in schwachem Alkohol oder F lemmingschem Gemisch fixierte Schnitte mit dem von Daddi zur Fettfärbung empfohlenen "Sudan III" gefärbt.

Bei dieser Methode wurde eine konzentrierte Lösung von Sudan III in $90 \%$ Alkohol zur Färbung benützt.

In diesen "xanthomähnlichen Zellen" waren ebenso wie im subkutanen Zellgewebe die Fettröpfchen intensiv rot gefärbt.

Aus diesen Reaktionen ging in eindentiger Weise hervor, daß die in diesen Zellen dicht aneinander gedrängten Tröpfchen aus F e t t bestehen.

Bei Übersichts- und Zellfärbungen der in A $1 \mathrm{kohol}$ fixierten und gehärteten Schnitte erscheinen diese Zellen mattgrau gefärbt, deutlich konturiert und an Stelle der Fettröpfohen treten Vacuolen auf, die der einzelnen Zelle ein schaumiges Gefüge, eine honigwabenähnliche 
Struktur verleihen, entsprechend dem Umstande, daß die Fettröpfehen durch die Konservierung gelöst sind.

Auf das Wesen dieser Zellen, sowie ihre Bedeutung, komme ich weiter unten noch zurück, da dieselben für die Auffassung des histologischen Bildes maßgebend sind.

Mit diesem Bilde, das in seinen Grundrissen aus verschieden langen, hypertrophierten, sich in die Tiefe der Coriumschichte einsenkenden interpapillären Retezapfen, in die Papillen sowie subpapilär eingelagerten "xanthomähnlichen Zellen “ besteht, verbindet sich innig ein zweites, welches dem lange Zeit bestehenden. hauptsächlich durch die in der Achselhöhle vorhandene Reibung und starke Schweißsekretion bedingten, chronischen Entzündungsprozesse seine Entstehung verdankt.

Mit diesem Entzündungsprozeß geht aber in allen Gewebsschichten ein ziemlich starkes $\ddot{O} \mathrm{dem}$ einher, welches sowohl zellige, als auch bindegewebige Elemente auflockert und in unregelmäßiger Weise auseinander drängt.

Zwischen den durch das Ödem auseinander gedrängten Bindegewebsbündeln verlaufen die ebenfalls auseinander gedrängten ela sti s chen F a sern, welche sich nur in der subpapillären Schichte finden und nicht in die einzelnen Papillen aufsteigen.

In der Cutis, sowie in den tieferen Schichten derselben sind die Blutgefäße beträchtlich erweitert. Die Gefäßwände selbst sind etwas verdickt, die Endothelzellen gequollen und springen stark in das Gefäßlumen vor.

Auch die $\mathrm{L} y \mathrm{mph}$ e fä $\mathrm{Be}$ sind mitbeteiligt, sie klaffen und zeigen eine stärkere Wand.

Am stärksten macht sich der Entzündungsprozeß entlang dem Verlaufe der Blut- und Lymphgefäße geltend. In engem Anschlusse an diese bilden die Infiltrationszellen in der subpapillären Schichte, weniger den Papillen kleine Haufen und Gruppen; seltener sind sie vereinzelt gelagert. Diese Zellen setzen sich aus mono- und polynucleären Leukocyten und aus Plasmazellen zusammen.

Die in der subpapillären Schichte gelegenen Zelihaufen steigen, von den Retezapfen durch einen Saum von Bindegewebe geschieden, in den Papillarkörper auf.

Die auch ziemlich reichlichen Mastzellen sind in den Papillen größtenteils randständig gelagert, weniger im subpapillären Bindegewebe und in den tieferen Schichten der Cutis, die Plasmazellen mehr in der Nähe der GefäBe sowie in der Subcutis.

In den durch das Ödem auseinander gedrängten Spalten der Stachelzellenschichte lagern sich bei dem bestehenden Entzündungsprozesse 
sowohl mono- als auch polynucleäre Leukocyten in kleineren Gruppen oder auch vereinzelt ein.

Die "xanthomähnlichen Ze]len" und die Infiltrationszellen stehen in einem umgekelirten Verhältnis zu einander. An denjenigen Stellen, sei es in der Cutis oder den Papillen, an denen der Infiltrationsproze $B$ überwiegt, treten die xanthomähnlichen Zellen an Zahl zurück und dort, wo der Enizündungsprozeß geringer erscheint oder ganz fehlt, sind die „xanthomäbnlichen Zellen" in größerer Anzahl oder so dicht aneinander gedrängt zu finden, daß sie die Papillen ganz ausfüllen und die Kouturen der einzelnen Zellen abflachen.

Die Schweißdrüsen sind, bei Berücksichtigung ihrer schon physiologiseh größeren Entwicklung in der Achselhöhle, ebenfalls etwas erweitert. Die Schweißdrüsenzellen zeigen außer einer geringen Vergrößerung jene degenerativen Veränderungen, welche schon physiologisch bei der Bildung des Schweißdrüsensekretes beobachtet werden.

Was die Stachelzellenschichte betrifft, so ist noch zu bemerken, daß die Stachel- oder Riffelzellen schon bei den gewöhnlichen Übersichtsfärbungen sehr deutlich auftreten.

Die Epithelfasern der Stachelzellenschichte sind mit geringen Unterbrechungen, die sich wohl auf das starke Ödem zurückführen lassen, überall sehr deutlich zu verfolgen. Die Verbindung der Basalzellenschichte mit der Stachelschichte ist durch die Epithelfasern eine sehr innige, während die von der Basalschichte in das papilläre und subpapilläre Bindegewebe ausstrahlenden Fasern nur an wenigen Stellen zu erkennen sind.

Die aus der rechten Achselhöhle excidierten Lymphdrüsen waren etwas vergrößert, von elastischer Konsistenz, auf dem Durchschnitt zeigten sie entsprechend ihrem chronisch-entzündlichen Zustande einen rötlich-gelben Farbenton.

Bei der histologi s chen Unter suchung derselben fiel zunächst die ziemlich beträchtliche Schwellung der Lymphfollikel auf, in letzteren Keimzentren mit zahlreichen Mitosen; ferner finden sich die Lymphsinus erweitert, ebenso auch die in den Balken verlaufenden kleinen Blutgefäße.

Das histologische Bild der mitverhornten Massen bedeckten, strichförmig verlaufenden $4-5 \mathrm{~cm}$ langen Naevi lineares zeigte ebenfalls eine mäßige Vergrößerung der Stachelzellenschichte, ähnlich verzweigt und verästelt, wie es das histologisehe Bild des Naevus verrucosus aus der Achselböhle darbietet.

In der subpapillären Cutisschichte ist ein geringes Infiltrat, aus mono- und polynucleären Leukocyten bestehend, sichtbar, welches in die Papillen zarte Ausläufer entsendet.

Der Stachelzellenschichte lagern sich verhornte Massen auf, die zum Teil mit einander innig zusammenhängen, zum Teil nur ganz locker sich aneinander lagern und der darunter liegenden Stachelzellenschichte auch nur in losem Zusammenhange stehen. 
Wie ich oben bereits erwähnte, stimmt die histologische Untersuchung des himbeerartigen Tumors an der Endphalange des kleinen Fingers der rechten Hand mit dem Bilde eines spitzen Kondyloms vollkommen überein.

Solche Wucherungen der obersten Hautschichten mit sehr früh ausgesprochener Neigung zur Papillombildung kommen fast ausschließlich an der Haut und Schleimhaut der Genitalorgane und in ihrer Nachbarschaft vor, extragenital sind dieselben seltener.

In der Literatur werden einige Fälle von extragenitalem Sitze spitzer Kondylome mitgeteilt, bei denen jedoch eine gonorrhoische Infektion vorlag.

Solche Fälle berichten Thévenin, Grünfeld, Gemy u. a.

Die spitzen Kondylome kommen nicht bloß bei Erwachsenen, sondern auck bei Kindern, die nicht gonorrhoisch erkrankt sind, noch waren, vor.

Einzelne Beobachtungen von Gemy, Ducrey und Oro, Rasch sprechen für die infektiöse Natur dieser Neubildungen, doch sind sie jedenfalls aur in geringem Maße kontagiös und die sehr wahrscheinliche Hypothese, dab es sich auch hier um spezifische Infektionserreger bandelt, beruht, wie Neisser hervorhebt, bisher mehr auf Analogieschlüssen und allgemein pathologischen Erwägungen, als auf positiven Tatsachen, da die Inokulationsversuche in größerem Umfange nicht geglückt sind.

In unserem Falle bestand bei dem 8jährigen Mädchen weder Fluor, noch Gonorrhoe, welche als ätiologisches Moment hätten herangezogen werden können. Vielmehr müssen wir für die Genese dieses himbeerförmigen 'Tumors am kleinen Finger der rechten $\mathrm{Hand}$ anhaltende entzündliche Reizungszustände - bestand ja doch auch ursprünglich eine Entzündung - für die Hypertrophie des Papillarkörpers, wobei die entzündlich infiltrierten Papillen mit ihren Gefäßen auswachsen und sich liäufig auch in $Z$ weige teilen, verantwortlich machen.

Die histologische Untersuchung der aus der A chselhöhle excidierten Stücke des Naevus verrucosus ergab durch die in den Papillen und im subpapillären Bindegewebe vorhandenen xanthomähnlichen Zellen insoferne eine Komplikation, als dieselbe - scheinbar - einen Widerspruch zwischen klinischer Beobachtung und histologischem Befund herbeiführte. 
Dieser, wenn auch scheinbare Widerspruch fordert die Beantwortung zweier Fragen.

Handelt es sich im vorliegenden Falle auf Grund des klinischen und histologischen Befundes um ein Xanthomatuberosumoderumeinenweichen, nicht pigmentierten Naevus verrucosus?

Zur Beantwortung dieser Fragen und einwandsfreien Beurteilung des histologischen Bildes wurden eine Reihe von Präparaten eines $X$ anthomatuberosum, sowie harter pigmentierter und weicher nichtpigmentierter Naevi untersucht.

In den Präparaten von Xanthoma tuberosum konnte die außerordentliche Ähnlichkeit der Xanthomzellen mit den in Frage stehenden xanthomähnlichen Zellen des $\mathrm{Naevus}$ verrucosus nicht von der Hand gewiesen werden.

In den Präparaten des $\mathrm{Naevus}$ verrucosus pigmentosus und Naevus pigmentosus et pilosus fielen sofort außer der starken Pigmentierung die für den Naevus charakteristischen Naevuszellen auf, die protoplasmareiche Zellen in Form von Haufen und Strängen in der Cutis darstellen.

Derartige Zellen, sowie Melanoblasten, die histologischen Merkmale eines weichen und pigmentierten Naevus waren in meinem Falle nicht aufzufinden.

AnStelle der Naevuszellen finden sich in den Schnitten des Naevus verrucosus außer den oben beschriebenen Infiltrationszellen, diesexanthomähnlichen Zellen in Anordnung und Gruppierung von Naevuszellen, während sich die für das Xanthoma tuberosum charakteristischen Xanthomzellen in kleineren oder größeren läppchenförmigen Herden gruppieren.

Für die Deutung des histologischen Befundes ist dieser Umstand wohl geeignet, diese xanthomähnlichen Zellen wegen der Übereinstimmung in Anordnung und Gruppierung mit Naevuszellen als Naevuszellen aufzufassen und auf Grund dieser Annahme schon durch die histologische Untersuchung allein, die Diagnose eines weichen, nicht pigmentierten Naevus verrucosus zu stïtzen. 
Zur Histologie dieses Falles müssen noch einige die Bedeutung dieser xanthomähnlichen Zellen erläuternde Worte hinzugefügt werden.

Bei schwacher Vergrößerung (ZeiB, Ocul. 2, Obj. $A A$ ) sieht man in den Präparaten mit Übersichtsfärbungen in den Papillen und subpapillär uur die Kerne der xanthomähnlichen Zellen mit verschiedener Deutlichkeit, nicht aber ihre Struktur, die erst bei starkerVergrößerung (Zeiß, Ocul. 2, Obj. $D D$ oder Öl-Immersion) deutlich zu Tage tritt.

Bei Anwendung $\mathrm{sch}$ wacher oder mittelstarker Vergrößerungslinsen wird eben die feinere Struktur, das schaumige, honigwabenähnliche Gefiige des Zellprotoplasmas nicht sichtbar, sondern nur deren Kerne.

Anf einen histologischen Unterschied sei noch besonders hingewiesen.

Während die Xanthomzellen des Xanthoma tuberosum auch in die tieferen Schichten der Cutis eindringen, liegen die xanthomähnlichen Zellen des Naevus verrucosus nur im Papillarkörper und in den obersten Teilen der Cutis.

Hinsichtlich der Histogenese der Xanthomzellen laben die Arbeiten von Virchow, Waldeyer, Chambard, $\mathrm{deV}$ in c enti is u. a. die Abstammung derselben von Bindegewebszellen hinreichend sichergestellt; de Vincentiis sieht in ihnen Endothelzellen des veränderten Bindegewebes, Tou to n und $\mathrm{Knaul}$ bezeichnen sie genauer als Abkömmlinge der Lymphspaltenendothelien, die ja dasselbe sind, wie die fixen Bindegewebszellen, nur daß sie - abweichend von der Norm die Fähigkeit haben, Fett zu bilden. Das entspricht der von Hallopeau und Török gewähiten Bezeichnung "heterotopische", „embryonale" Fettzellen.

Es steht auf Grundlage der Arbeiten von Waldeyer, Virchow, Touton, 'Török u. a. außer Zweifel, daß die Fetteinlagerung die eigentümliche Gelbfärbung der verschiedenen Xanthomformen bedingt und auch darüber stimmen die Anschauungen der meisten Autoren überein, daß die Fetteinlagerung nicht die Folge eines degenerativen Vorganges sei. 
Die Xanthomzelle ist also der charakteristische Bestandteil des Xanthoms, sowohl des flachen, als auch des knötchenförmigen, doch erscheint dieselbe in letzterem meist geringeren Umfanges als jene im Xanthom der Augenlider.

An osmierten Präparaten fällt vor allem das Vorhandensein mehr oder weniger läppchenförmiger, schwarzer Einlagerungen in der Cutis auf, welche aber von der Epidermis durch einen deutlichen Saum normaler oder nur wenig veränderter Cutis getrennt erscheinen.

Die schwarzen Massen erweisen sich als ein Konglomerat grauer bis schwarzgrauer Schollen, welche gegen die Mitte ziemlich dicht gedrängt stehen und kaum ein Zwischengewebe erkennen lassen, während sie gegen die Peripherie lockerer gefügt, von faserigem Bindegewebe durchsetzt erscheinen.

An Präparaten, an denen das Fett mittelst Alkohols oder Äther extrahiert wurde, erweisen sich die an Osmiumpräparaten erscheinenden schwarzen Schollen als mehr oder weniger umfangreiche, ein oder mehrkernige Zellen, welche nach der Beschreibung der meisten Autoren, ron einem sehr feinen, protoplasmatischen Netze, welches runde oder fast runde Maschen einschließt, erfüllt sind.

Neben den verschieden geformten und großen, mehrkernigen Zellen des Xanthoms kommen auch eigentümliche (Touton, Unna) vor, deren Kerne um ein trübes Zentrum in regelmäßigem Kreise, der noch von einem breiten Saume von Protoplasma umgeben ist, gestellt erscheinen.

Die Beschreibung dieser Xanthomzellen stimmt vollständig mit den in meinem Falle als "xanthomähnliche" Zellen bezeichneten überein, die nur infolge ihrer analogen Anordnung und Gruppierung die Rolle der Naevuszellen über$\mathrm{n}$ e h m en.

Der Einwand, daß die in Rede stehenden xanthomähnlichen Zellen keine Xanthomzellen, sondern Naevuszellen mit epithelialer Abkunft im Sinne Unnas seien, bedarf der Widerlegung.

Judalewitsch führt in seiner jüngst erschienenen Arbeit „Zur Histogenese der weichen Naevi" ans, daß die aus dem Epithel ausgestoßenen Zellen sich nach und nach verändern, allmählich das Pigment 
verlieren, eine runde Form annehmen, durchsichtig werden, indem ihr Protoplasma ein feines, zartes Reticulum darstellt, in welchem ein dunkel tingierter Kern liegt.

Die Zellen sind in diesem Stadium der Umwandlung nach $J$ udalewitsch den Talgdrüsenzellen überraschend ähnlich. Dieses Stadium, in welchem die Zellen die Form und das Aussehen der Talgdrüsenzellen annehmen, scheint nach $J$ udalewitsch für die Entwicklung der Naevi unentbehrlich zu sein.

Bevor ich die Argumente anführe, welche nach meinen Untersuchungen gegen die epitheliale Abkunft der xanthomähnlichen Zellen als Naevuszellen sprechen, seien die Merkmale der Talgdrüsenzellen an dieser Stelle besonders hervorgehoben.

Es erscheint mir dies aus dem Grunde nicht unwichtig, weil einerseits die Talgdrüsenzellen nach der Auffassung von Hebra, später Geber und O. Simon mit dem Xanthom insoferne in Beziehung gebracht wurden, als die genannten Autoren das Xanthom als den Ausdruck einer "hyperplastischen Entwicklung von Talgdrüsenzellen" auffaßten, andererseits nach den Ausführungen von Judalewitsch u. a. diese Zellformen nur Übergangsstadien der Naevuszellen bei ihrer Entwicklung aus den Epithelzellen darstellen.

Diese Talgdrüsenzellen sind nach der Beschreibung von Geber und Oskar Simon große, platte, entweder rundliche oder durch gegenseitige Abplattung polygonale Zellen. Der Kern, welcher meist zentral gelagert ist, ist an allen Zellen deutlich sichtbar und zeichnet sich meist durch ein stärkeres Lichtbrechungsvermögen aus. Der Zellkörper zeigt an einzelnen Stellen die Konturen kleiner, rings um den Kern gestellter Fettropfen, häufiger aber einen granulierten, feinkörnigen Inhalt.

Diese Beschreibung genügt schon, um darzutun, daß die Talgdrüsenzellen wohl eine gewisse Ähnlichkeit mit den Xanthomzellen besitzen, aber in ihrem Aussehen doch so wesentliche Unterschiede in der Größe und Kontur der Zelle, Lage des Kernes, Struktur des Stroma, Anordnung der Fettröpfchen aufweisen, daß schon histologisch die Abstammung der Xanthomzelle von den Talgdrïsenzellen in Frage gestellt wird.

Was nun die epitheliale Abkunft der Naevuszellen, somit auch der als Übergangsstadium bezeichneten talgdrüsenähnlichen Zelle anlangt, so fand ich in meinen Präparaten an keiner Stelle einen unmittelbaren Zusammenhang des Deckepithels mit den xanthomähnlichen Zellen. Selbst an den Stellen, an denen die Stränge von xanthomähnlichen Zellen nahe an die Reteschicht herankamen, war ein deutlicher Zwischenraum, der beide Zelllagen von einander trennte, wahrzunehmen. 
Ich bin also nicht in der Lage, die Ansichten von Unna, Abesser, Kromayer, Delbanco, Hodara, Herrmann, Magnus, Möller u. a. zu bestätigen. ${ }^{\text {) }}$

Ebenso konnte ich an keiner Stelle eine Abschnürung, als ob die Zellen oder Zellgruppen aus dem Epithel in das Bindegewebe herausfallen oder "abtropfen", konstatieren.

Einem anderen Einwande, daß in diesem Falle ein Degenerationsproze $\beta$, eine fettige Degeneration der Naeruszellen in diesexanthomähnlichenZellen vorliegt, kann ich vom pathologisch-anatomischen Standpunkte aus widerlegen.

Gegen diese Annahme eines Degenerationsprozesses spricht auf Grund des mikroskopischen Befundes die Tatsache, daß nicht in einem einzigen der Präparate ein Anfangs., Übergangs- oder Endstadium ron Naevuszellen in die xanthomähnlichen Zellen aufzufinden war.

Diese besitzen mit Xanthomzellen eine unverkennbare Ähnlichkeit, nicht aber mit den Talgdrüsenzellen im Sinne vоц Judalewitsch, die ein Übergangsstadium der Naevuszellen aus den Epithelzellen darstellen.

Diese xanthomähnlichen Zellen ahmen nur durth ihre Lage und Anordnung in Haufen und Strängen, und nicht in läppchenförmigen Herden wie beim Xanthoma tuberosum, den Typus ron Naevuszellen nach.

Der Naevus war nicht pigmentiert, daher konnte über das Verhalten des Pigmentes nichts weiteres mitgeteilt werden.

Histologisch lag nun aus folgenden Gründen die Berechtigung vor, diesen wegen der xanthomähnlichen Zellen bemerkenswerten Fall den Naevi beizuzählen.

1) Anmerkung bei der Korrektur: E. Riecke gelangt in seiner Arbeit "Zur N a evu sfrage" (Arch. f. Dermatol. u. Syph. Bd. LXV. 1903) zu dem Sehlusse, daß die Naevuszellen mit den Bindegewebszellen der embryonalen Cutis in genetischem Zusammenhang stehen und daß die Anordnung der Naevuszellen den Zwischenräumen des Bindegewebes entspricht. Bilder, welche als Abtropfungserscheinungen vom Epithel gedeutet werden könnten, entstehen durch Andrängen der Naevusmassen gegen die Epidermis. 
Außer der zirmiich bedeutenden $\mathrm{Ak}$ a $\mathrm{n}$ those ist besonders die Hypertrophie des Papillarkörpers hervorzuheben. Durch die Einlagerung der xanthomähnlichen Zellen in den Papillarkörper wird der Typus eines weichen und nicht pigmentierten Naevus vollständig gewahrt.

Nach dem makroskopischen Aussehen, wie auch mikroskopischen Bilde ist dieser Naevus als ein Naevus verru. cosus zu bezeichnen, der weich und nicht pigmentiert ist, seiner Struktur nach den Gewebsnaevi beigezählt werden muß.

Die zahlreichen Herde von Infiltrationszellen sind durch den akzessorischen Entzündungsprozeß, der hauptsächlich auf eine mechanische Irritation des Naevus zurückzuführen ist, hinreichend erklärt.

Aulor den Argumenten der histologischen Untersuchung spricht klinisch gegen die Diagnose eines Xanthoma tuberosum zunächst die Lokalisation.

Als Prädilektionsstellendesknötchenförmigen $\mathrm{X}$ anthoms sind insbesondere die Druckstellen zu verzeichnen.

So finden sich die einzelnen Knötchen besonders an den Ellenbogen, den Knieu, über den Fingergelenken und ad nates, in der Kreuzbeingegend ror; sie lokalisieren sich aber auch an der Flachhand, der Fußsohle und an beliebigen Körperstellen, sells:t am behaaiten Kopf.

Das Xanthoma tuberosum unterscheidet sich aber auch durch die Form und Farbe ron dem Naevus verrucosus. Das knötchenförmige Xinthom erscheint zumeist disseminiert und generalisiert, zeichnet sich in der Regel durch die Bildung hirsekorn- bis erbsengroßer, bald weicher, bald derberer und selbst knorpelharter, kugeliger oder ovaler, leicht drüsiger Knötchen von strohgelber bis gelblichbrauner Färbung aus, welche von einer unveränderten Oberha u t überkleidet sind.

Weiters ist zu bemerken, daß das Xanthoma tuberosum im Kindesalter selten, vom 30. Lebensjahre an viel häufiger vorkommt. 
Nicht nur die klinische Beobachtung und der histologische Befund, sondern auch die Genese selbst bietet uns einige Anhaltspunkte, welche die Kombination eines Xanthoms und Naevus auch rom ätiologischen Standpunkte aus rechtfertigen.

Unna hebt zwar hervor, daß die Zellinfiltration des gewöhnlichen Xanthoms der Augenlider nicht die mindeste Analogie, ja nicht einmal eine entfernte Ähnlichkeit mit der der Naevi besitzt.

$\mathrm{Chambard}$ bezweifelte die Einheitlichkeit der Xanthome und behauptete, daß die xanthomatöse Zelldegeneration sich in den verschiedensten Neubildungen finde, und daß es $n$ eb en einem reinen Xanthome auch xanthomatöse Mischgeschwülste gäbe.

Nach Hallopeau müssen die Xanthome zu den Naevi gezählt werden, mit denen sie sowohl die Struktur, als gewisse klinische Merkmale teilen. Hallopeau weist auf die Tatsache hin, daß Xanthome öfters auf Naevi angetroffen werden, und erwähnt eines Falles, wo zahlreiche Xanthome linienförmig und dem Verlauf eines Nerven entsprechend angeordnet waren. Ihr spätes Auftreten spreche nicht gegen diese Ansicht, indem auch die Naevi öfters erst beim Erwachsenen auftreten.

Ebenso wie Hall opeau, halten anch Tout on, Köbner, K. Her $x$ heimer die Xanthome für Neubildungen embryonalen Ursprungs, d. h. für Naevi, da sie dieselbe Anordnung haben und in analoger Weise entsprechend der Verbreiterung der Hautnerven auftreten können.

Chauvière neigt ebenfalls der Annahme zu, daß das gewöhnliche Xanthom eine gutartige Neoplasie embryonalen Ursprungs sei, analog den Naevi.

Auch Ziegler ist der Ansicht, daß das Xanthom nahe mit dem Naevus pigmentosus verwandt ist und betrachtet als den einzigen wesentlichen Unterschied rom pathologisch-anatomischen Standpunkte die Fetteinlagerung in die zu Nestern oder Strängen gruppierten, neugebildeten Zellen.

Die Frage der Heredität in der Ätiologie der Xanthome ist bereits von vielen Beobachtern hervorgehoben worden. 
Alle diese Tatsachen, wie Heredität, Vorhandensein bei der Geburt oder Auftreten in früher Jugend, die Kombination mit Naevi, Anordnung wie systematisierte Naevi, sprechen dafür, daß es sich beim Xanthom um Neubildungen a uf kongenitaler Grundlage handelt.

Daher erscheint es notwendig, diesen Naevus von der großen Naevusgruppe abzusondern, selbständig zu rubrizieren und als „xanthomartigen Naerus" zu bezeichnen.

Alle diese obenangeführten Tatsachen lassen sich ebenso gat auf den xanthomartigen Naevus verrucosus der Achselhöhle anwenden, indem wir uns die Kombination des Naerus mit den eingelagerten xanthomähnlichen Zellen als eine Neubildung im Sinne der Cohnheimschen Theorie der Geschwulstentwicklung aus embryonalen Keimanlagen entstanden, ungezwungen erklären.

Die in unserem lialle strichförmig verlaufenden, mit verhornten Massen bedeckten, an Fingern, Zehen und ad nates lokalisierten Gebilde gehören ebenfalls zur großen Gruppe der "Naevi" und werden allgemein als "Naeri lineares" bezeichnet.

Die Bezeichnung „Naevus line aris" präjudiziert nichts, sie ist nur als Ausdruck für alle diejenigen naevusartigen Gebilde gewählt, welche in Linien und Streifen angeordnet sind und welche gewisse feste Richtungen an der Haut einhalten, so daß alle Neubildungen dieser Art ä u $ß$ e r $l i c h$ den verschiedensten Anblick darbieten, bloß durch die topographische Übereinstimmung innerhalb jeder Region sich ähnlich sehen.

Diese Gebilde haben seit jeher die Aufmerksamkeit auf sich gelenkt und sind je nach der Auffassung des ätiologischen, klinischen oder histologischen Momentes unter den manuigfaltigsten Namen beschrieben worden.

Bärensprung glaubte, analog wie beim Herpes zoster in dieser Affektion den Ausdruck einer angeborenen Erkrankung der Spinalganglien erblicken zu sollen und benante dieselbe "Naevus unius lateris", während Th. Simon die vermutete Beziehung zu den nervösen Einflüssen durch die Bezeichnung „Nervennaevus", Gerhardt durch jene ${ }_{n}$ neuropathisches Hautpapillom" zum Ausdrucke brachte.

Ebenso bat sich auch Spietschk für die neurotrophische Natur der linearen Naevi im Sinne Bärensprungs ausgesprochen. 
Die ursprünglich auf rein äußerliche Momente basierte Auffassung von Bärensprung, Th. Simon, Gerhardt u. a. hat eine Reihe von Bedenken wachgerufen, welche der Annahme der nervősen Natur der Naevi lineares entgegenstehen und dieselben auf Entwicklungskomplikationen zurückgeführt.

Philippson hat auf die Kongruenz der Naevusstreifen mit den Voigtschen Grenzlinien der Nervenbezirke hingewiesen, während Blaschko in dem Hervorsprossen neuer, histologiseh differenter Gebilde die Grundlage der Differenzierangsbezirke erblickt.

Jaddassohn hat auf Grundlage der Zusammenstellung einer größeren Reihe von $\mathbf{F}$ ällen gezeigt, daß es eine Gruppe solcher gibt, deren Ver. teilung keine Übereinstimmung mit den Voigtschen Nervengrenzlinien aufweist, dagegen eine Kongruenz mit den Haarströmen und den ron Voigt zu ihrer Charakterisierung gezeichneten Linien zeigt.

Pečirka, Hallopeau und Weil u. a. haben auf die Kongruenz der Verteilung mancher systematisierter Naevi am Rumpf mit den metameralen, den einzelnen Rückenmarkssegmenten entsprechenden Gebieten hingewiesen, und damit zugleich die Annahme, daß die Naevi Entwicklungskomplikationen ihre Entstehung verdanken, gestützt.

Neuerdings unternahm Meissner den Versuch, die zentralnervöse Aatur der Naevi zu vertreten und Heller glaubt eine Übereinstimmung mit den Lymphgefäßen der Haut nachweisen zu können.

Jadassohn hat im Anschlusse an Besnier und Hallopeau die hierhergehörigen Formen, für welche die Bezeichnung der Naevi lineares nicht ausreicht, unter dem Namen der ns stematisierten. $\mathrm{Naevi“} \mathrm{zusammengefaßt} \mathrm{und} \mathrm{zunächst} \mathrm{alle} \mathrm{strichförmigen} \mathrm{Erkrankungen}$ den Naevi zuzuweisen, d. h. auf embryonale Veranlagung zurückzufübren gesucht.

Blaschko glaubt nicht, daß es nötig ist, von der Bezeichnung „lineäre oder streifenförmige Naevi abzugehen, da die streifenförmige Anorảnung derselben selbst da, wo fleckförmige und flächenhafte Gebilde daneben bestehen, doch immer das im Krankheitsbilde ansschlag. gebende ist. Freilich gilt das nur für eine ganz eng begrenzte Gruppe von Naevi, die sogenanten verrucösen oder ichthyotischen (Unnas harte) Naevi.

Eine Reihe von Beobachtungen linearer Erkrankungen mit dem mehr oder weniger ausgesprochenen Charakter bestimmter Dermatonosen (Neurodermitis linearis chronica Touton, Lichen ruber LederinannMeyer, ekzemähnliche Erkrankungen Shearar, Unna, Sterntbal u. a.) haben besonders Touton und Ledermann-M e yer zu der Schlußfolgerung geführt, daß, so berechtigt die Beziehung gewisser strichförmiger Erkrankungen (der wahren sogenannten Nervennaevi) auf entwicklungsgeschichtliche (embryonale) Störungen auch sein mag, es doch nicht angehe, diesen Entwicklungsmodus für alle linearen Erkrankungen in Anspruch zu nehmen, beziehungsweise die embryonale 
Grundlage aus der streifenförmigen Anordnung zu erschließen, nachdem es sich in den angezogenen Fällen ja um entzündliche Erkrankungen handle.

Bertamini nahm in seiner jüngst erschienenen Arbeit "Zur Kenntnis der strichförmigen Erkrankungen "Gelegenheit, an der Hand eines Falles die Kasuistik dieser Erkrankungen zu erweitern.

Die Aktivität des Prozesses, welche sich sowohl im klinischen Verlaufe als im histologischen Befunde kundgab, rechtfertigt es, wie Bertamini ausführt, seinen Fall in die Kategorie der linearen Dermatonosen und nicht der $\mathrm{Naevi}$ einzureiben.

Auch Unna hat auf Grund seiner Beobachtungen eine Reihe von Einwänden erboben, nach welchen alle Neubildungen dieser Art trotz ihrer Unterschiede wegen der gleichen topographischen Verhältnisse zum Verwechseln ähnlich sehen und diese naevusäbnliche Affektion durch ihren akuten Beginn, die entzündlichen Perioden und den Wechsel des äußeren Habitus Züge darbietet, welche sonst den Naevi im allgemeinen fremd sind.

U u na gelangt zu dem Schlusse, daß bei der Gruppe der „N a evi lineares “ trutz der frappanten Analogie in topographischer Beziehung sowohl klinisch, wie histologisch die größten Differenzen bestehen.

Mit Bezug auf die sogenannte Ichthyosis circumscripta hält Jadassohn diese für eine auf kongenitaler Anlage beruhende reine Verhornungsanomalie und von den Naevi zu scheiden ist, die immer, auch wenn sie noch so ausgebreitet sind, cirkumscript sind und bei denen die Mischung von verschiedenen Tumorformen mit Hyperkeratosen in der Tat sehr häufig ist, während bei der Ichthyosis die kutane Tumorbildung fehlt.

Kaposi rechnet die als Ichthyosis hystrix beschriebene Affektion, der böchste Grad der Ichthyosis, ebenfalls den Naevi zu; dem entgegen bemerkt jedoch $J$ a dassohn, daß in den Fällen diese in Linien und Streifen scharf begrenzt auftritt, sie zu den Naevi gehört.

Aus der großen I ülle der hierher gehörigen, in der Literatur mitgeteilten Fälle seien nur wenige angeführt.

J. Neumann fand bei einem 6 Tage alten kräftigen Kinde einen Naevus papillaris, der ad nates rechterseits und an der rechten unteren Extremität in regelmäßig angeordneten Linien und Streifen lokalisiert war.

Kaposi beobachtete einen Fall von Naevus unius lateris, der vom Handteller ausgehend, sich einerseits gegen den Ulnarrand bis zur Mitte des Vorderarmes, andererseits über die Mitte der Beugeseite der Finger in linearer Anordnung sich fortsetzte. Dieselben Erscheinungen fanden sich am inneren Fußrand, dem Dorsum der großen Zehe, Sprunggelenk bis in die Malleolargegend.

Einen ähnlichen Fall beschrieb derselbe Autor als $\mathrm{N}$ aevus verrucosus an der Beugeseite der Finger, bei dem vom Netacarpus angefangen scbmutzigbraune, harte Auflagerungen an beiden Händen, sowie lateralwärts an der Fubsohle auftraten. 
Galewski und Schlossmann teilen die Krankengeschichte eines Falles von "Naevus linearis" mit, dessen Verlauf den Voigtschen Grenzlinien an rechten Oberschenkel entsprach.

Eine Reihe klinisch ähnlicher Beobachtungen, welche in den angeführten Fällen stets die Anordnung von Linien und Streifen zeigen, an den verschiedensten Körperstellen lokalisiert, in jhrer feineren Struktur entweder verrucösen oder papillomatösen Bau darbieten, liegen von Buri, A. Alexander, Spi egelberg, Pringle, P. A. Morrow, P. Meissner u. a. vor.

Hinsichtlich der elementaren pathologischen Formen, welche diese Naevi zusammensetzen, ist hervorzuheben, daß sie eine große Mannigfaltigkeit darbieten können. Erscheinen sie zumeist in Gestalt der harten Naevi, also als mit mehr oder weniger dunkel gefärbter, abkratzbarer Hornschichte bekleidete Erhebungen, so nehmen sie in anderen Fällen, oder an verschiedenen Stellen desselben Falles die Form von papillären Exkreszenzen an, oder sie erscheinen bald da, bald dort als weiche Warzen oder glatte Hyperpigmentationen.

Noch mannigfaltiger als das klinische Bild kann sich das histologis che gestalten.

Seitdem wir nun wissen, daß die Naevi klinisch und anatomisch keine einheitlichen Gebilde darstellen, ist der Begriff des $\mathrm{Na}$ evas, der ein ätiologischer ist, für die Nomenklatur nicht zu entbehren.

Es haftet dem Namen Naevus der Begriff des Ange. borenseins untrennbar an, aber es gibt auch Fälle, bei denen nur die Anlage kongenital besteht, während sich die Affektion erst später, bis zur Zeit der Pubertät entwickelt.

Wenn wir nun die in unserem Falle, namentlich an den Fingern und Zehen vorhandenen, mit Schuppen und Hornmassen bedeckten, linear verlaufenden Wucherungen zu den Naevi rechnen, so sind es folgende Momente, welche diese Angliederung an die $\mathrm{Naevusgruppe}$ und $\mathrm{Klassifizierung}$ als "Naevi lineares" rechtfertigen.

Zunächst handelt es sich um eine kongenitale Affektion, zumindestens eine solche, die wenige Tage nach der Geburt auftrat und durch ihre wesentlich strichförmige Anordnung, die ganz besondere Neigung eine verru- 
cöse Oberfläche bald mit stärkerer, bald mit geringerer Verhornung zu bilden ausgezeichnet ist. An einzelnen Stellen konnte ein spontaner Abfall von Schuppen, nie aber eine spontane Rückbildung des in Rede stehenden Naevus beobachtet werden.

Nach genauester Berücksichtigung aller klinischen Symptome, sowie des histologischen Bildes sind in unserem Falle lineare Erkrankungen mit dem mehr oder weniger ausgesprochenen Charakter bestimmter Dermatonosen mit Sicherheit auszuschließen.

Was die entzündlichen Erscheinungen bei dieser Form der Naevi anlangt, so hat Jadassohn besonders hervorgehoben, daß es abgesehen von den Fällen, bei denen Mazeration und Reibung vielfach zur Exsudation und Ulzeration Anlaß gibt, noch eine ganze Reihe von Fällen übrig bleibt, bei denen das entzïndliche Element, o th ne da $B$ äu $ß$ e re Momente mit Sicherheit als Ursache dafür anzuführen wären, in einer auffallenden Weise in den Vordergrund tritt.

Übereinstimmend mit unseren histologischenUntersuchungen sind als die histologisch herrorstechendsten Momente der strichförmigen Muttermäler Hyperkeratose und Akanthose mitverschiedenartiger Gestaltung des Papillarkörpers anzuführen und müssen wir in unserem Falle diese Naevi lineares zu den Gewebsnaevi rechnen.

Die bei den Naevilineares ron vielen Autoren angeführten, häufig vorhandenen Sensibilitätsstörungen konnten in unserem Falle nicht konstatiert werden, wohl aber ein gewisser Grad von Atrophie der Muskulatur der rechten oberen Extremität.

$\mathrm{Ob}$ und inwieweit nun für das Zustandekommen dieser linearen Naevi eine vorausgegangene Erkrankung des Nervensystems bezw. einzelner Metameren derselben anzunehmen ist. vermag ich nicht zu entscheiden, jedenfalls ist eine solche, wie Blaschko in den Schlußsätzen seines am VII. Kongresse der deutschen dermatologischen Gesellschaft erstatteten Referates hervorhebt, nicht mit Notwendigkeit anzunehmen. 


\section{Schlußsätze.}

1. Der himbeerförmige Tumor am kleinen Finger der rechten Hand gleicht sowohl seiner äußeren Konfiguration, als auch seiner Struktur nach vollkommen dem eines spitzen Kondyloms. Für die Genese dieses Tumors müssen wir anhaltende entzündliche Reizungszustände verantwortlich machen.

2. Der Naevus verrucosus der rechten Achselhöhle ist ein weicher, nicht pigmentierter Gewebsnaevus, der durch die Einlagerung von xanthomähnlichen Zellen in die Papillen und das subpapilläre Bindegewebe als "xanthomartiger Naevus" bezeichnet werden muß. Diese xanthomähnlichen Zellen ahmen nur durch ihre Anordnung und Gruppierung den Typus von Naevuszellen nach, sie sind keine Degenerationsprodukte im Sinne einer fettigen Degeneration, auch nicht epithelialer Abkunft im Sinne der Unnaschen Naevuslehre. Die Genese dieser xanthomähnlichen Zellen glauben wir ungezwungen, nach der Co h n h e i mschen Theorie der Geschwulstentwicklung aus embryonalen Keimanlagen entstanden, erklären zu können.

3. Die an den Fingern und Zehen, ad nates mit verhornten Massen bedeckten, strichförmig verlaufenden Naeri lineares sind als harte Naevi zu bezeichnen.

Histologisch zeichnen sich dieselben durch eine Hyperkeratose und Akanthose mit verschiedenartiger Gestaltung des Papillarkörpers aus.

Die linearen Naevi sind die Folge von Entwicklungsstörungen, für deren Zustandekommen eine vorausgegangene Erkrankung des Nervensystems nicht mit Notwendigkeit anzunehmen ist.

Zum Schlusse gestatte ich mir, meinem hochverehrten Lehrer Herrn Geheimrat Prof. Dr. A. Neisser für die gütige Überweisung des Falles und den Hinweis auf seine Bedeutung, Herrn Prof. Dr. R. Paltauf für die liebenswürdige Unterstützung und vielfachen Ratschläge, sowie Herrn Assistenten Dr. R. Maresch meinen besten Dank auszusprechen.

Herrn Prof. Dr. G. Riehl sage ich für die besondere Liebenswürdigkeit der Durchsicht meiner Präparate, sowie der mir erteilten Ratschläge vielen Dank. 


\section{Liter atur.}

1. A besser, M. Über die Herkunft und Bedeutung der in den sogenannten Naevi der Haut vorkommenden Zellhaufen. Virchow s Arch. Bd. CLXVI. pag. 40.

2. Alexander, A. Ein Fall von Naevus linearis unius lateris. Derm. Zeitsehrift. Bul. II.

3. Beck, Cornelius. Ein Fall von Naevus papillomatosus universalis. Monatsheft f. prakt. Dermatol. Bd. XXXII. 1901.

4. Bendal, H. Sur un cas de dégénérescence xanthomateuse. Thèse de Paris 1895. Ref. im Zentralblatt für allgem. Pathologie und patholog. Anatomie, 1896.

5. Bertamini, J. Zur Kenntuis der strichförmigen Erkrankungen. Arch. f. Derm. und Syph. Bd, LXIr.

6. Blaschko. Über Naevus. Berl. dermat. Gesellsch. 1895, in Arch. f. Derm. und Syph. 1895. Bd. IIL.

7. Blasehko. Die Nervenverteilung in der Haut in ihrer Be. ziehung zu den Erkrankungen der Haut (mit ausführlicher Literatur). VII. Kongreß der deutsch. dermatol. Gesellschaft. Breslau. 1901.

8. Chambard. Dernières recherches anat. et chir. sur le Xauthome. Annales. 1882.

9. Chambard et Guillard. Nouvelle contribut. à l'ćtude des transformat. des tumeurs congénitales de la peau. Annales. 1883.

10. Chauvière, J. Contribution à l'étude de la forme juvénile du Xanthome. Thèse de Paris 1895. Ref. im Zentralblatt f. allg. Pathologie und pathol. Anatomie. 1895.

11. Delbanco, E. Epithelialer Naevus. Monatshefte für prakt. Dermat. Bd. XXII.

12. I) crey und Oro. Beitrag zur pathol. Histologie, Ätiologie und Pathogenese des Condylom. acumin. Ref. in Monatsbefte für prakt. Dermatol. 1892. I.

13. Galewski und Schlossmann. Über Naevus linearis. Deutsch. Arch. für klin. Medizin. 1896.

14. Geber, E. und Simon, Oskar. Zur Anatomie des Xanthoma palpebrarum. Arch. für Derm. und Syph. 1872.

15. Gemy. Über extragenitale Condylom. acuminata. Monatshefte für prakt. Dermatol. 1894, I. 
16. Geyer. Beitrag zur Histologie und Lebre vom Xanthoma tuberos. multiplex. Arch. für Dermat. und Syph. 1897. III.

17. Grünfeld, J. Zur Kasuistik der spitzen Kondylome. Arch. für Derm. und Syph. 1875.

18. Hallopeau. Sur la nature des xanthomes et la cause prochaine des leurs complications. La Médec. moderne. 1893.

19. Hallopeau. Les naevi. Progrès médic. Nr. 28. Ref. in Jahresbericht d. ges. Medizin. Virch ow-Hirsch. 1891. II.

20. Herrmann, H. Zur Naevusfrage. VII. Kongreß der deutsch. dermatol, Gesellschaft. Breslau. 1901.

21. Herxheimer, K, Neoplasmen der Haut. Lubarsch-O stertag, Ergebnisse d. spez. Pathol., Morphologie und Physiologie. 1896. 4.

22. Hodara, M. Das Verhalten der Epithelfaserung während der Entwickelung der weichen Muttermäler und des alveolären Carcinoms. Monatshefte für prakt. Dermatol. Bd. XXV.

23. J a d a s s o h $\mathbf{n}$ und Werner. Zur Kenntnis der systematisierten Naevi (mit ausführlicher Literatur). Arch. für Derm. und Syph. 1895.

24. Jaris ch, A. Die Hautkrankheiten, Nothnagel, Spez. Pathologie und Therapie.

25. Judalewitsch, G. Zur Histogenese der weichen Naevi. Arch. für Dermat. und Syph. Bd. LVIII.

26. Juli us berg, M. Zur Theorie der Pathogenese des spitzen Kondyloms. Arch. f. Derm. u. Syph. Bd. LXIV.

27. Kaposi. Pathologie und Therapie der Hautkrankheiten. 1899.

28. Kaposi. Ein Fall von Naevus verrucosus. Virchow-Hirs ch, Jahresbericht d. ges. Medizin. 1885. 2.

29. Kaposi. Ein Fall von Naevus unius lateris. Krank.-Demonstr. Wien. dermat. Gesellsch. im Arch. f. Derm. und Syph. 1896. Bd. XXXIV. 30. Kaposi. Fin Fall von Naevus verrucosus an der Beugeseite der Finger. Arch. für Derm. und Syph. 1897. 2.

31. Kroma yer, E. Zur Histiogenese der weichen Naevi. Metaplasie von Epithel zu Bindegewebe. Dermatol. Zeitschrift. 1896.

32. Löwenbach, G. Beitrag zur Histogenese der weichen Hautnaevi. Virchows Arch. CLXXV. Bd. 1899.

33. L ö en b a $\mathrm{h}, \mathrm{G}$, Über spitze Kondylome der Lippen und Mundschleimhaut. Festschrift Neumann 1900.

34. Meissner, P. Über Naevus verrucosus. Derm. Zeitschr. 1895.

35. Möller, Magnus. Naevusstudien. Arch. für Derm. und Syph. Bd. LXII.

36. Morrow, P. A. Zwei Fälle von strichförmigem Naevus. NewYork. med. Journal. 1898. Ref. in Monatshefte f. prakt. Derm. 1898. 1.

37. Morrow, P. A. Xanthoma tuberculatum, report of a case with remarks on a new and succes ful mode of treatment. Journ, of cut. and gén.-urin. Jan. 1893. Ref. in Arch. für Derm. u. Syph. 1894. 1.

38. Neisser-Jadassohn. Die Krankheiten der Haut in Schwalbe-Ebstein, Handbuch der prakt. Medizin. 
39. Ne umann, J. Über Naevus papillaris. Österr. Jahrbücher für Paediatrie. 1877. 2.

40. Neumann, J. Lehrbuch der Hautkrankheiten. 1880.

41. Pappenheim, A. Eine neue, chemisch-elektive Doppel. färbung für Plasmazellen. Monatshefte für prakt. Dermatologie. 1901. 2. 42. Pollitzer, S. The nature of the xanthomata. New-York. med. Journal. 1899 ,

43. Pranter, V. Zur Färbung der elastischen Fasern. Zentralblatt für allgem. Pathologie und pathol. Anatomie. Bd. XIII. 1902.

44. Pringle. Ein Fall von Naevus linearis papillomatosus. London. dermat. Gesellsch. 1898. Ref. im dermatol. Zentralblatt. 1899.

45. Rasch. Nosologische Bemerkungen über Kondylome. Derm. Zentralbl. 1900.

46. Riecke, E. Zur Naevusfrage. Arch. f. Dermat. u. Syphilis. Bd. LXV.

47. Rist, E. Naevi. La Pratique Dermatologique. Tom. III. p. 559.

48. Spiegelberg. Über einen Fall von angeborener papillomatöser, sogen. neuropathischer Warzenbildung. Münch. med. Wochenschr. 1896. Nr. 30 .

49. S pietsehka, Th. Über sogen. Nerven-Naevi. Arch. für Derm. and Syph. 1894.

50. Thévenin. Vegetations du prepuce et du cuir chevelu. Ref. im Arch. für Derm. und Syph. Bd. LII.

51. Török, L. Über die Natur des Xanthoms, nebst einigen kritischen Bemerkungen über das Wesen des Tumors. Ref. im Monatsh. für prakt. Dermat. 1894. 2.

52. Touton, Karl. Über das Xanthom, insbes. dessen Histologie und Histiogenese. Arch. für Derm. und Syph. 1885.

53. Unna. Histopathologie der Hautkrankheiten.

54. Ziegler, E. Lehrbuch der allgem. Anatomie und Pathogenese. Allgem. und spez. Teil. 


\section{Erklärung der Abbildungen auf Taf. VII u. VIII.}

Fig. 1. Der himbeerförmige Tumor an der Endphalange des kleinen Fingers der rechten Hand.

Fig. 2. Der xanthomartige Naevus verrucosus der r. Achselhöhle.

Fig. 3. Schnitt durch den xanthomartigen Naevus verrucosus der rechten Achselböhle. (Schwache Vergrößerung, $\mathrm{Z} \theta$ iss Okul. 2, Obj. A A.) Die hypertrophierten Papillen sind in verschiedener Richtung getroffen. Außer einer ziemlich stark entwickelten Akanthose, subpapillär in Haufen angeordneten Infiltrationszellen und erweiterten Gefäßen der Cutis und Subcutis, sind von den in die Papillen und subpapillär eingelagerten xanthomähnlichen Zellen bei schwacher Vergrößerung ( $Z$ e is s Okul. 2, Obj. $A A$ ) nur die Kerne dieser Zellen mit verschiedener Deutlichkeit sichtbar.

Fig. 4. Die in der vorigen Abbildung in der Mitte gelegene Papille mit starker Vergrößerung. (Z e is s Okul. 2, Obj. $D D$ oder ÖlImmersion.) Zwischen beiden Säulen der Stachelzellenschicht sind die xanthomähnlichen Zellen mit ihrem schaumigen, honigwabenähnlichem Protoplasmaleib, zentral oder exzentrisch gelegenem Kern sichtbar und zwischen diesen xanthomähnlichen Zellen eingestreut zahlreiche monound polynucleäre Leukocyten. 